\title{
Robust Analysis and Parameters Identification of the Nonlinear Multistage Dynamical System in Batch Fermentation of Glycerol
}

\author{
Xu Zhang, ${ }^{1}$ Xinying Wang, ${ }^{1}$ Enmin Feng, ${ }^{1}$ and Zhilong Xiu ${ }^{2}$ \\ ${ }^{1}$ School of Mathematical Science, Dalian University of Technology, Dalian, Liaoning 116024, China \\ ${ }^{2}$ Department of Biotechnology, Dalian University of Technology, Dalian, Liaoning 116012, China \\ Correspondence should be addressed to Xu Zhang; zhangxu0451@163.com
}

Received 18 June 2014; Accepted 3 September 2014; Published 21 October 2014

Academic Editor: Henggui Zhang

Copyright (c) $2014 \mathrm{Xu}$ Zhang et al. This is an open access article distributed under the Creative Commons Attribution License, which permits unrestricted use, distribution, and reproduction in any medium, provided the original work is properly cited.

\begin{abstract}
This paper discusses a complex biological problem which is the fermentation of glycerol by Klebsiella pneumoniae in batch culture. We set up an improved multistage model involving the concentration of intracellular substances. Furthermore, the existence, uniqueness, and continuity of solutions with respect to the parameters are discussed. On the condition that glycerol and 1,3propanediol are assumed to pass the cell membrane by passive diffusion coupled with facilitated transport, we take the relative errors between experimental data and computational values of the extracellular substances concentrations and the biological robustness of the intracellular substances concentrations as the performance index. Then we establish a parameter identification model and construct the particle swarm optimization algorithm to solve it. Finally, the numerical result shows that the improved model could describe the glycerol fermentation in batch culture well.
\end{abstract}

\section{Introduction}

1,3-Propanediol (1,3-PD) is an important chemical raw material with a wide range of applications, such as lubricants, antifreeze agents, solvents, adhesives, and laminates. In particular, 1,3-PD is the main raw material of PTT which is a new synthetic fibre with excellent performance. PTT can be used to produce the carpet, engineering plastics, garment material, and so forth. PTT was synthesized in 1941, but until 1998 it was produced on a large-scale due to the low production and high price of 1,3-PD in the past. The price of PTT is controlled by the price of $1,3-\mathrm{PD}$, so it is necessary to make the production of 1,3-PD higher and the price of 1,3-PD lower. Now there are two methods that produce 1,3-PD: one is chemical synthesis and the other is microbial fermentation. The microbial fermentation which is the bioconversion of glycerol to 1,3-PD has been paid much attention due to its low cost, high production, no pollution, and so forth [1-3]. However, its production of 1,3-PD is lower compared with the traditional chemical synthesis because of the immature technology. Hence, many researchers have tried to investigate the glycerol bioconversion to 1,3-PD by Klebsiella pneumoniae since 1980s [4].
In 1995, Zeng and Deckwer [5] proposed a five-dimensional dynamical system of glycerol fermentation, in which the concentrations of biomass, glycerol, and products (1,3$\mathrm{PD}$, acetate, and ethanol) in reactor were considered. Xiu and Zeng [6] and Gao et al. [7] did further research work for the five-dimensional dynamical system, including parameter identification, optimal control, and dynamic behaviour analysis. However, all of these researches did not consider the changes of the intracellular substances concentration.

In 2008, Sun et al. [8] firstly proposed a nonlinear dynamical system involving concentration changes of three intracellular substances (glycerol, 1,3-PD, and 3hydroxypropionaldehyde (3-HPA)) and two key enzymes (1,3-PD oxidoreductase (PDOR) and glycerol dehydratase $(\mathrm{GDHt}))$ in glycerol fermentation to $1,3-\mathrm{PD}$ by Klebsiella pneumoniae. In 2011, based on Sun's model, Wang et al. [9] inferred the possible ways across the cell membrane of glycerol and 1,3-PD. The conclusion is used in this paper.

Compared with continuous and feed-batch cultures, glycerol fermentation in batch culture can obtain the highest production concentration. So nonlinear dynamical system for this culture has been extensively considered in the paper of Gao et al. [10]. The typical cell growth in batch culture 
includes several growth phases, which are defined as the lag, exponential growth, decreased growth, and death phases. In 2009, Wang et al. [11] provided an improved model of glycerol fermentation in batch culture in order to describe the multistage features without considering the concentrations of the intracellular substances.

In this paper, we study the fermentation of glycerol covering both extracellular and intracellular environments. Furthermore, we apply the specific rate of cell growth mentioned in the paper of Song et al. [12] to the eightdimensional dynamical system which is nonautonomous, multistage, and nonlinear. In this way, we obtain a novel mathematical model concerning the glycerol fermentation in batch culture. Simultaneously, the Lipschitz and linear growth conditions are proved; furthermore, we discuss the existence and uniqueness of solutions to the system. Subsequently, due to the lack of experimental data about the concentrations of intracellular substances, we introduce the description of biological robustness which was described in words in the literature [13-15]. In this paper, we present the quantitative description of biological robustness in an unsteady process which is different from the one which is defined in a steady state in $[16,17]$. Taking the robustness of intracellular substance concentrations as the performance index in addition to the relative errors between experimental data and computational values of the extracellular concentrations, we establish a nonlinear dynamical model with 27 parameters to be identified and prove the identifiability of the model. At last, we construct a numerical algorithm based on the particle swarm optimization [18] and solve the identification model.

The paper is organized as follows. In Section 2, an eightdimensional nonlinear dynamical system of batch culture is presented. The existence, uniqueness, and the continuity of solutions are proved in Section 3. In Section 4, a quantitative description of the substances about the system robustness and the identification model is presented. A feasible algorithm is constructed and the optimum parameters are identified at the end of the paper.

\section{Nonlinear Dynamical System}

In order to describe the metabolic process in detail, Figure 1 gives the metabolic pathway of glycerol conversion into 1,3PD by Klebsiella pneumoniae.

During glycerol metabolism by Klebsiella pneumoniae under anaerobic conditions at $37^{\circ} \mathrm{C}$ and $\mathrm{pH}=7.0$, glycerol is first transported across the cell membrane from the extracellular environment to the intracellular environment, and then it is further catabolized, reactions catalyzed by enzymes, to generate intermediates and final products, for example, 3-hydroxypropionaldehyde (3-HPA), 1,3-PD, acetic acid, ethanol, and so forth. Finally, the products are transported across the cell membrane from the intracellular environment to the extracellular environment. As shown in Figure 1, the transport mechanisms of glycerol and 1,3-PD

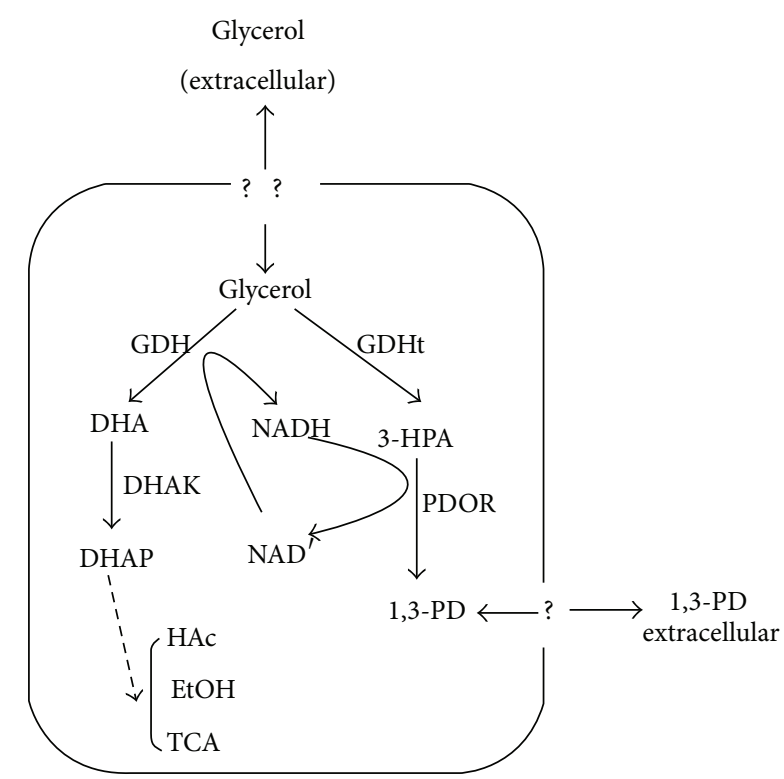

FIGURE 1: The metabolic pathway of glycerol converts into 1,3-PD by Klebsiella pneumoniae. Abbreviations: GDHt, glycerol dehydratase; 3-HPA, 3-hydroxypropionaldehyde; 1,3-PD, 1,3propanediol; PDOR, 1,3-PD oxidoreductase; GDH, glycerol dehydrogenase; DHA, dihydroxyacetone; DHAK, dihydroxyacetone kinase; DHAP, dihydroxyacetonephosphate; HAc, acetic acid; EtOH, ethanol; TCA, TCA cycle.

across the membrane have not been observed in experiments yet. Glycerol and 1,3-PD may pass the membrane by both passive diffusion and active transport or by passive diffusion only. In this paper, we use the conclusion which has been inferred in [9].

According to the work [9], on the assumption that both glycerol and 1,3-PD pass the cell membrane by active transport coupling with passive diffusion under substratesufficient conditions, the nonlinear dynamical system of glycerol batch culture fermentation can be described by

$$
\begin{gathered}
\dot{x}_{1}(t)=\mu x_{1}, \\
\dot{x}_{2}(t)=-q_{2} x_{1}, \\
\dot{x}_{3}(t)=q_{3} x_{1}, \\
\dot{x}_{4}(t)=q_{4} x_{1}, \\
\dot{x}_{5}(t)=q_{5} x_{1}, \\
\dot{x}_{6}(t)=\frac{1}{k^{7}}\left(k^{8} \frac{x_{2}}{x_{2}+k^{9}}+k^{10}\left(x_{2}-x_{6}\right)\right. \\
\left.\times N_{+}\left(x_{2}-x_{6}\right)-q_{20}\right)-\mu x_{6},
\end{gathered}
$$




$$
\begin{gathered}
\dot{x}_{7}(t)=\frac{k^{11} x_{6}}{K_{m}^{G}+K_{m}^{G}\left(x_{7} / k^{12}\right)+x_{6}} \\
-\frac{k^{13} x_{7}}{K_{m}^{P}+x_{7}\left(1+x_{7} / k^{14}\right)}-\mu x_{7}, \\
\dot{x}_{8}(t)=\frac{k^{13} x_{7}}{K_{m}^{P}+x_{7}\left(1+x_{7} / k^{14}\right)} \\
-\frac{k^{15} x_{8}}{x_{8}+k^{16}}-k^{17}\left(x_{8}-x_{3}\right) N_{+}\left(x_{8}-x_{3}\right)-\mu x_{8},
\end{gathered}
$$

where

$$
N_{+}(\xi)= \begin{cases}1, & \xi \geq 0 \\ 0, & \xi \leq 0\end{cases}
$$

$x(t)=\left(x_{1}(t), \ldots, x_{8}(t)\right)^{T}$, respectively, denotes the concentrations of biomass, extracellular glycerol, extracellular 1,3-PD, acetic acid, ethanol, intracellular glycerol, 3-HPA, and intracellular 1,3-PD at time $t$ in reactor and the unit is $\mathrm{mmolL}^{-1}$. Besides, $K_{m}^{G}=0.53 \mathrm{mmol} / \mathrm{L}$ and $K_{m}^{P}=$ $0.14 \mathrm{mmol} / \mathrm{L}$ are Michaelis-Menten constants.

On the basis of [9], the specific cell growth rate of biomass (i.e., $\mu$ ) is expressed by the following equation:

$$
\mu=\mu_{m} \frac{x_{2}}{x_{2}+K_{s}}\left(1-\frac{x_{2}}{x_{2}^{*}}\right)\left(1-\frac{x_{3}}{x_{3}^{*}}\right)\left(1-\frac{x_{4}}{x_{4}^{*}}\right)\left(1-\frac{x_{5}}{x_{5}^{*}}\right) .
$$

In the above equation, $\mu_{m}$ is the maximum specific growth rate and the value is $0.67 \mathrm{~h}^{-1}$ and $K_{s}=$ $0.28 \mathrm{mmolL}^{-1}$ is Monod saturation constant for substrate. $x_{2}^{*}=2039 \mathrm{mmolL}^{-1}$ denotes the maximum residual substrate concentration and $x_{i}^{*}(i=3,4,5)$ denotes the maximum product concentration whose values are $939.5 \mathrm{mmolL}^{-1}$, $1026 \mathrm{mmolL}^{-1}$, and $360.9 \mathrm{mmolL}^{-1}$, respectively.

However, the typical cell growth in batch culture includes several growth phases, which are defined as the lag, exponential growth, stationary growth, and death phases. We can easily find that the value of $x_{2}$ will decrease below zero using (2). Therefore, it cannot predict the stationary growth and death phase. Based on the above analysis, we will modify (10) by the form given in the literature [12] as follows:

$$
\begin{aligned}
\mu= & \mu_{m} \exp \left(\frac{-\left(t-t_{m}\right)^{2}}{2 t_{l}^{2}}\right) \frac{x_{2}}{x_{2}+K_{s}}\left(1-\frac{x_{2}}{x_{2}^{*}}\right) \\
& \times\left(1-\frac{x_{3}}{x_{3}^{*}}\right)\left(1-\frac{x_{4}}{x_{4}^{*}}\right)\left(1-\frac{x_{5}}{x_{5}^{*}}\right),
\end{aligned}
$$

where $t_{l}$ is the starting moment of lag growth phase and $t_{m}$ is the time when $\mu$ reaches the maximum. Let $I:=\left[0, t_{f}\right]$ denote the total fermentation time. According to the actual experiments, it is easy to know $0 \leq t_{l} \leq t_{m} \leq t_{f}$.
Considering the intracellular environment as a black box model, the specific consumption rate $q_{20}$ of extracellular glycerol is defined as

$$
q_{20}=m_{2}+\frac{\mu}{Y_{2}}+\Delta q_{2} \frac{x_{2}(t)}{x_{2}(t)+K_{2}^{*}} .
$$

According to the literature [9], extracellular glycerol and 1,3-propanediol are assumed to pass the cell membrane by passive diffusion coupled with facilitated transport, so the specific consumption rate of substrate can be expressed by

$$
q_{2}=k^{1} \frac{x_{2}}{x_{2}+k^{2}}+k^{3}\left(x_{2}-x_{6}\right) N_{+}\left(x_{2}-x_{6}\right) .
$$

The specific formation rate of extracellular 1,3-PD is

$$
q_{3}=k^{4} \frac{x_{8}}{x_{8}+k^{5}}+k^{6}\left(x_{8}-x_{3}\right) N_{+}\left(x_{8}-x_{3}\right) .
$$

The specific formation rates of acetate and ethanol can be expressed by (refer to [10])

$$
\begin{aligned}
& q_{4}=m_{4}+\mu Y_{4}, \\
& q_{5}=m_{5}+\mu Y_{5} .
\end{aligned}
$$

In (1)-(15), $k^{1}, \ldots, k^{17}, m_{2}, m_{4}, m_{5}, Y_{2}, Y_{4}, Y_{5}$, and $\triangle q_{2}$ are the parameters which are needed to be identified.

Therefore, the nonlinear dynamical system of glycerol fermentation in batch culture can be described as follows:

$$
\begin{aligned}
\dot{x}(t) & =f(t, x, u), \\
x(0) & =x_{0},
\end{aligned}
$$

where $x_{0}$ denotes the initial state. Consider

$$
f(t, x, u):=\left(f_{1}(t, x, u), \ldots, f_{8}(t, x, u)\right)^{T} .
$$

$f_{i}(t, x, u), i \in I_{8}$, denote the right-hand side of the $i$ th equation of (1)-(8).

Since the concentrations of extracellular and intracellular material are restricted in a certain range according to the practical production, denote the admissible set of $x$ by $W_{\text {ad }}:=$ $\prod_{i=1}^{8}\left[x_{i *}, x_{i}^{*}\right]$, where $x_{i *}$ and $x_{i}^{*}$ indicate allowable lower and upper bounds of state variable $x(t)$, respectively. The admissible set of $u$ is denoted by $U=\prod_{i=1}^{27}\left[u_{i *}, u_{i}^{*}\right]$, where $u=\left(k^{1}, \ldots, k^{17}, m_{2}, m_{4}, m_{5}, Y_{2}, Y_{4}, Y_{5}, \triangle q_{2}, K_{2}^{*}, t_{l}, t_{m}\right)^{T} \in$ $R^{27}$ represent the kinetic parameters. According to [9], the boundary of $u$ is listed as shown in Table 1 .

\section{The Properties of the Nonlinear Dynamical System}

In this section, we study the questions of existence, uniqueness, and continuity of solutions with respect to the parameters.

According to the factual experiments, we make the assumptions as shown in Table 2. 
TABLE 1: The boundary of $u_{i *}$ [9].

\begin{tabular}{lcccccccc}
\hline$u_{1 *}$ & $u_{2 *}$ & $u_{3 *}$ & $u_{4 *}$ & $u_{5 *}$ & $u_{6 *}$ & $u_{7 *}$ & $u_{8 *}$ & $u_{9 *}$ \\
\hline 30.0 & 1.0 & 100.0 & 60.0 & 1.0 & 50.0 & 5.0 & 40.0 & 0.5 \\
\hline$u_{10 *}$ & $u_{11 *}$ & $u_{12 *}$ & $u_{13 *}$ & $u_{14 *}$ & $u_{15 *}$ & $u_{16 *}$ & $u_{17 *}$ & $u_{18 *}$ \\
\hline 100.0 & 1.0 & 100.0 & 1.0 & 0.01 & 0.5 & 1.0 & 1.0 \\
\hline$u_{19 *}$ & $u_{20 *}$ & $u_{21 *}$ & $u_{22 *}$ & $u_{23 *}$ & $u_{24 *}$ & $u_{25 *}$ & $u_{26 *}$ & $u_{27 *}$ \\
\hline-1.455 & 2.61 & 0.0041 & 16.535 & 5.83 & 14.29 & 5.715 & 0.0 & 3.0 \\
\hline
\end{tabular}

TABLE 2: The boundary of $u_{i}^{*}[9]$.

\begin{tabular}{lcccccccc}
\hline$u_{1}^{*}$ & $u_{2}^{*}$ & $u_{3}^{*}$ & $u_{4}^{*}$ & $u_{5}^{*}$ & $u_{6}^{*}$ & $u_{7}^{*}$ & $u_{8}^{*}$ & $u_{9}^{*}$ \\
\hline 70.0 & 5.0 & 5000.0 & 150.0 & 10.0 & 100.0 & 10.0 & 70.0 & 3.0 \\
\hline$u_{10}^{*}$ & $u_{11}^{*}$ & $u_{12}^{*}$ & $u_{13}^{*}$ & $u_{14}^{*}$ & $u_{15}^{*}$ & $u_{16}^{*}$ & $u_{17}^{*}$ & $u_{18}^{*}$ \\
\hline 5000.0 & 50.0 & 300.0 & 50.0 & 2.0 & 20.0 & 30.0 & 100.0 & 3.3 \\
\hline$u_{19}^{*}$ & $u_{20}^{*}$ & $u_{21}^{*}$ & $u_{22}^{*}$ & $u_{23}^{*}$ & $u_{24}^{*}$ & $u_{25}^{*}$ & $u_{26}^{*}$ & 3.5 \\
\hline-0.485 & 7.83 & 0.0123 & 49.605 & 17.49 & 42.87 & 17.145 & 6.0 \\
\hline
\end{tabular}

(H1) The concentration of reactants are uniform in reactor; while time delay and non-uniform space distribution are ignored.

(H2) No medium is pumped inside and outside the bioreactor in the process of batch fermentation.

To begin with, we discuss some properties of the function $f(t, x, u)$.

Property 1. For fixed $u \in U$, the function $f(t, x, u)$ defined by (17) satisfied that

(i) $f$ is locally Lipschitz continuous in $x$ on $W_{\text {ad }} \subseteq R^{8}$;

(ii) $f$ satisfies linear growth condition in $x$; that is, there exists a constant $0<k<\infty$, such that, for all $x \in W_{\text {ad }}$,

$$
f(t, x, u) \leq k(\|x\|+1),
$$

where $\|\cdot\|$ is Euclidean norm.

Proof. (i) Suppose that the functions $h(x)$ and $l(x)$ are as follows:

$$
\begin{aligned}
& h(x):=\left(x_{2}-x_{6}\right) N_{+}\left(x_{2}-x_{6}\right), \\
& l(x):=\left(x_{8}-x_{3}\right) N_{+}\left(x_{8}-x_{3}\right) .
\end{aligned}
$$

Now we show that $h(x)$ and $l(x)$ are Lipschitz on the set $W_{\text {ad }} \subseteq$ $R^{8}$. below.

First, for all $x, y \in W_{\text {ad }}$, four kinds of cases are discussed

(1) When $x_{2}-x_{6} \geq 0$ and $y_{2}-y_{6} \geq 0$, we have that $h(x)=x_{2}-x_{6}$ and $h(y)=y_{2}-y_{6}$; then

$$
\begin{aligned}
\|h(y)-h(x)\| & =\left\|y_{2}-y_{6}+x_{6}-x_{2}\right\| \\
& \leq\left\|y_{2}-x_{2}\right\|+\left\|y_{6}-x_{6}\right\| \\
& \leq 2\|y-x\| .
\end{aligned}
$$

(2) When $x_{2}-x_{6} \geq 0$ and $y_{2}-y_{6}<0$, we have that $h(x)=x_{2}-x_{6}$ and $h(y)=0$; then

$$
\begin{aligned}
\|h(y)-h(x)\| & =\left\|x_{2}-x_{6}\right\| \\
& <\left\|x_{2}-x_{6}-\left(y_{2}-y_{6}\right)\right\| \\
& \leq\|y-x\| \leq 2\|y-x\| .
\end{aligned}
$$

(3) When $x_{2}-x_{6}<0$ and $y_{2}-y_{6} \geq 0$, we have that $h(x)=0$ and $h(y)=y_{2}-y_{6}$; then

$$
\begin{aligned}
\|h(y)-h(x)\| & =\left\|y_{2}-y_{6}\right\| \\
& <\left\|y_{2}-y_{6}-\left(x_{2}-x_{6}\right)\right\| \\
& \leq\|y-x\| \leq 2\|y-x\| .
\end{aligned}
$$

(4) When $x_{2}-x_{6}<0$ and $y_{2}-y_{6}<0$, we have that $h(x)=0$ and $h(y)=0$; then

$$
\|h(y)-h(x)\|=0 \leq 2\|y-x\|
$$

Let $\alpha=2$; then

$$
\|h(y)-h(x)\| \leq \alpha\|y-x\|, \quad \forall x, y \in W_{\mathrm{ad}} .
$$

That is, the function $h(x)$ is Lipschitz on the set $x \in W_{\text {ad }}$.

It can be proved in a similar way that $l(x)$ in $x \in W_{\text {ad }}$ is locally Lipschitz continuous. Hence, in view of the definition of $f(t, x, u), f(t, x, u)$ is Lipschitz continuous in $x \in W_{\text {ad }}$.

(ii) Because of the actual glycerol metabolism by Klebsiella pneumoniae, all the substances' concentrations are in 
the fixed range; there exist constants $M_{1}, M_{2}$, such that $\left|x_{2}(t)-x_{6}(t)\right|<M_{1},\left|x_{8}(t)-x_{3}(t)\right|<M_{2}, t \in \mathscr{F}$. Let

$$
\begin{aligned}
& S_{1}:=\left|m_{2}\right|+\mu_{m}\left|\frac{1}{Y_{2}}\right|+\left|\Delta q_{2}\right|, \\
& S_{2}:=\left|k_{1}\right|+\left|k_{3}\right| M_{1}, \\
& S_{3}:=\left|k^{4}\right|+\left|k^{6}\right| M_{2}, \\
& S_{4}:=\left|m_{4}\right|+\mu_{m}\left|Y_{4}\right|, \\
& S_{5}:=\left|m_{5}\right|+\mu_{m}\left|Y_{5}\right|,
\end{aligned}
$$

for $\left|x_{2} /\left(x_{2}+K_{2}^{*}\right)\right|<1$; it follows that

$$
\left|q_{20}\right| \leq S_{1}, \quad\left|q_{i}\right| \leq S_{i}, \quad i=2,3,4,5 .
$$

Let $L_{1}:=\mu_{m}$ and $L_{i}:=S_{i}, i=2,3,4,5$; then

$$
\begin{gathered}
\left|f_{1}\right|=|\mu|\left|x_{1}\right| \leq \mu_{m}\left|x_{1}\right| \leq L_{1}(\|x\|+1), \\
\left|f_{2}\right|=\left|q_{2}\right|\left|x_{1}\right| \leq S_{2}\left|x_{1}\right| \leq L_{2}(\|x\|+1), \\
\left|f_{i}\right|=\left|q_{i}\right|\left|x_{1}\right| \leq S_{i}\left|x_{1}\right| \leq L_{i}(\|x\|+1), \quad i=3,4,5 .
\end{gathered}
$$

Let $S_{6}:=\left(\left|k_{8}\right|+\left|k_{10}\right| M_{1}+S_{2}\right) /\left|k_{7}\right|$ and $L_{6}:=\max \left\{C_{6}, \mu_{m}\right\}$, and then

$$
\begin{aligned}
\left|f_{6}\right| & \leq \frac{1}{\left|k_{7}\right|}\left(\left|k_{8}\right|+\left|k_{10}\right|\left|x_{2}-x_{6}\right|+\left|q_{20}\right|\right)+|\mu|\left|x_{6}\right| \\
& \leq S_{6}+\mu_{m}\|x\| \leq L_{6}(\|x\|+1) .
\end{aligned}
$$

Let $S_{7}:=\left|k_{11}\right|+\left|k_{13}\right|$ and $L_{7}:=\max \left\{S_{7}, \mu_{m}\right\}$, and then

$$
\begin{aligned}
\left|f_{7}\right| & \leq S_{7}+|\mu|\left|x_{7}\right| \\
& \leq S_{7}+\left|\mu_{m}\right|\|x\| \leq L_{7}(\|x\|+1) .
\end{aligned}
$$

Let $S_{8}:=\left|k_{13}\right|+\left|l_{1} k_{15}\right|+\left|l_{2} k_{17}\right| M_{2}, L_{8}:=\max \left\{S_{8}, \mu_{m}\right\}$, and

$$
\begin{aligned}
\left|f_{8}\right| & \leq\left|k_{13}\right|+\left|l_{1} k_{15}\right|+\left|l_{2} k_{17}\right|\left|x_{8}-x_{3}\right|+|\mu|\left|x_{8}\right| \\
& \leq S_{8}+\mu_{m}\|x\| \leq L_{8}(\|x\|+1) .
\end{aligned}
$$

Denote $L=\max \left\{L_{i}, i \in I_{8}\right\}$ and $k=2 \sqrt{2} L$; then we have $\|f(t, x, u)\| \leq k(\|x\|+1)$.

Definition 1. Suppose that $x_{0} \in W_{\text {ad }}$ and $u \in U$, the set of admissible initial-state-control pairs $\left(x_{0}, u\right)$ is denoted by $H:=W_{\text {ad }} \times U$, and $x\left(\cdot ; x_{0}, u\right)$ is said to be a solution of (16), if it satisfies the following integral equation:

$$
x\left(t ; x_{0}, u\right)=x_{0}+\int_{0}^{t} f(s, x, u) d s, \quad t \in I .
$$

Property 2. For any $\left(x_{0}, u\right) \in H$, the system (16) has a unique solution $x\left(\cdot ; x_{0}, u\right) \in C_{b}\left(I, R^{8}\right)$ and $x\left(\cdot ; x_{0}, u\right)$ is continuous in $u$ on $U$.

Proof. Firstly, it is easy to prove the existence of the solution. Then we will prove that the system (16) has a unique solution.
Suppose that $x_{1}\left(\cdot ; x_{0}, u\right)$ and $x_{2}\left(\cdot ; x_{0}, u\right)$ are two solutions of system (16); it follows from formula (31) and the fact that $f$ is Lipschitz continuous in $x$ that

$$
\begin{aligned}
& \left\|x_{1}\left(t ; x_{0}, u\right)-x_{2}\left(t ; x_{0}, u\right)\right\| \\
& \quad \leq \int_{0}^{t}\left\|f\left(s, x_{1}, u\right)-f\left(s, x_{2}, u\right)\right\| d s \\
& \quad \leq L \int_{0}^{t}\left\|x_{1}\left(s ; x_{0}, u\right)-x_{2}\left(s ; x_{0}, u\right)\right\| d s, \quad \forall t \in I .
\end{aligned}
$$

By Bellman Gronwall inequality, it is proved that $\left\|x_{1}\left(\cdot ; x_{0}, u\right)-x_{2}\left(\cdot ; x_{0}, u\right)\right\|_{c}=0$; that is, system (16) has a unique solution.

By Definition 1, it is easy to know that the solution $x\left(\cdot ; x_{0}, u\right)$ is continuous. At last, applying the classical theory of differential equation, we can easily obtain the continuity dependence of the solution on the parameter vector $u$.

\section{Biological Robustness Analysis and Identification Model}

In order to evaluate the reliability of system (16), we should give an evaluation criterion. The real data of extracellular substances has been measured by some experiments. In a general way, the computational results should be in accordance with experimental data. So we construct an identification model in which $u \in U$ are the parameters to be identified.

To identify the optimal parameters, we give two performance indexes. One is the relative errors between experimental data and computational values of the extracellular concentration and the other is the biological robustness. Robustness is one of the fundamental properties of biological system. Then we will present two definitions: one is the relative error of extracellular substances and the other is the relative deviation with respect to parameters perturbation. To begin with, on the basis of the actual experiment, we make the following assumptions:

(H3) given $x_{0} \in W_{\text {ad }}$, system (16) is controllable and observable;

(H4) the set $U$ is nonempty in $R^{27}$.

Definition 2. Let $y_{i}\left(t_{j}\right)$ and $x_{i}\left(t_{j}, u\right), i \in I_{3}$, be the $i$ th experimental extracellular concentration and the computational value at the $t_{j}, j \in I_{7}$ moment. Then, the relative error of extracellular substances' concentration (i.e., biomass, glycerol, and 1,3-PD) is defined as

$$
\operatorname{SSD}(u):=\frac{1}{3} \sum_{i=1}^{3} \sum_{j=1}^{7} \frac{\left(x_{i}\left(t_{j}, u\right)-y_{i}\left(t_{j}\right)\right)^{2}}{7\left(y_{i}\left(t_{j}\right)\right)^{2}} .
$$

In the above equation, 3 denotes three extracellular substances (i.e., biomass, glycerol, and 1,3-PD). Since the feeding of alkali into the reactor during the fermentation process, whose purpose is to maintain the $\mathrm{pH}$ value at 7.0 or so, it leads to the inaccuracy of the extracellular concentrations of acetic acid and ethanol. This paper is concerned with the relative 
error between experimental data and computational values of the first three substances. $t_{1}=1, t_{2}=2, t_{3}=3, t_{4}=4, t_{5}=5$, $t_{6}=6$, and $t_{7}=6.92$ are the measurement time points of the extracellular concentration in the experimental process.

Because of the current experiment conditions, the intracellular material concentrations are commonly difficult to measure accurately. On the other hand, the main purpose of the model concerned with the intracellular concentration is for the explanation of biological mechanisms to understand the metabolic processes. Therefore, the intracellular model should consider some basic characteristics of the biological system.

The quantified biological robustness is a feasible method to evaluate the validity of the computational concentrations of intracellular substances. Robustness is one of the fundamental characteristics of biological systems and it is a property that allows a system to maintain its functions against internal and external perturbations [19]. Perc and Marhl defined biological robustness, which is usually evaluated by the sensitivity analysis [20] and frequency [21], via a given parameter and a dependant variable. With increasing interest in system biology, robustness has attracted serious scientific research [22-24]. However, the quantitative descriptions of the biological system's robustness and the corresponding mathematical models concerning concentration changes of intracellular substances are seldom found. The definition of biological robustness for batch culture should meet the case: for the overall process of batch culture, the changes of state variables provoked by parameters distribution are smaller and the system's performance is more robust [22]. Consequently, owing to that the state variables of the system are the important factors to determine the system's main function, we define the distribution level as the robustness of the system after the disturbance of $u \in U$. The robustness of the system (16) is as follows.

Definition 3. For any $u \in U$, suppose that $B(u, \delta):=\{v \mid$ $\|v-u\| \leq \delta\}$ is the disturbance neighborhood of $u(\delta>0)$; then for any $v \in B(u, \delta)$, the relative deviation with respect to parameter $u$ is defined as

$$
\operatorname{MSD}(u, v):=\sum_{i=6}^{8} \int_{0}^{t_{f}} \frac{\left|x_{i}(t, u)-x_{i}(t, v)\right|}{\left|x_{i}(t, u)\right|} d t
$$

Definition 4. For any $u \in U$, suppose that $B(u, \delta):=\{v \mid$ $\|v-u\| \leq \delta\}$ is the disturbance neighborhood of $u(\delta>0)$; we extract randomly $n_{p}$ sampling points following the uniform distribution. The distribution set is $\left\{v_{j} \mid v_{j} \in B(u, \delta), j \in I_{n_{p}}\right\}$. Then the average relative deviation with respect to parameters $u$ is defined as

$$
\operatorname{MSD}_{\mathrm{av}}(u):=\frac{1}{n_{p}} \sum_{j=1}^{n_{p}} \sum_{i=6}^{8} \int_{0}^{t_{f}} \frac{\left|x_{i}(t, u)-x_{i}\left(t, v_{j}\right)\right|}{\left|x_{i}(t, u)\right|} d t
$$

Based on the previous study the parameter identification model can be formulated as follows:

$$
\begin{aligned}
& \text { IP: } \\
& \min \quad J(u):=\operatorname{SSD}(u)+\operatorname{MSD}_{\mathrm{av}}(u) \\
& \text { s.t } \quad u \in U, \quad \dot{x}=f(t, x, u), \quad x \in W_{\mathrm{ad}}, t \in\left[0, t_{f}\right] .
\end{aligned}
$$

Now, we define the set $S$ in order to discuss the following problem easily, given that $x_{0} \in W_{\text {ad }}, S:=\left\{x\left(\cdot ; x_{0}, u\right) \mid\right.$ $x\left(\cdot ; x_{0}, u\right)$ is the solution of the system (16), and $x\left(\cdot ; x_{0}, u\right) \in$ $\left.W_{\text {ad }}, u \in U\right\}$.

Lemma 5. Given $x_{0} \in W_{a d}$, the set $S$ defined above is compact on $C\left(I, R^{8}\right)$.

Theorem 6. There exists an optimal solution of IP; that is, there exists an $u^{*} \in U$ such that

$$
J\left(u^{*}\right) \leq J(u), \quad \forall u \in U
$$

Proof. It follows from Property 2 that the mapping from $u \in U$ to $x\left(\cdot ; x_{0}, u\right) \in S$ is continuous. Therefore, $J(u)$ is continuous in $u$. By the compactness of $S$, we can conclude that (IP) has an optimal solution, denoted by $u^{*}$ such that

$$
J\left(u^{*}\right) \leq J(u), \quad \forall u \in U .
$$

\section{Particle Swarm Optimization Algorithm and Numerical Results}

Since IP would be hard to solve directly and it contains a huge number of numerical computations of differential equations and judgments of the approximately stable solutions, solving the whole IP is intolerable for a personal computer. In other words, it is impossible to work out within an acceptable time. Therefore, we construct the improved particle swarm optimization algorithm.

Remark 7. In order to achieve the following algorithm, we calculate the formula (34) as follows approximatively:

$$
\operatorname{MSD}(u, v) \approx \sum_{i=6}^{8} \sum_{j=1}^{m} \frac{\left|x_{i}(h h * j, u)-x_{i}(h h * j, v)\right|}{\left|x_{i}(t, u)\right|} * h h .
$$

Here, we use the thought of definite integral and segment the interval $\left[0, t_{f}\right]$ into $m=t_{f} / h h$ parts, where $h h$ is the step size. Furthermore, we take the right endpoint as the approximate value and hence we get the above equation.

Algorithm 1. Particle swarm optimization algorithm.

Step 1 (initialization). The number of particles is denoted by num, learning factors are $c 1, c 2$, the maximum and minimum inertia weights are $w s$, we, the maximum iteration is $T_{\max }$, 
and the iterations are $m$. Let $m=1$; the control factors are $d 1$, $d 2$, the inertia weight is $w$, the search scope of the particle is $U \in R^{27}$, the individual optimal value of the particle is pbest, the optimization position of the particle is pbest $_{u}$, the group optimal value is $g$ best, and the group optimal position of the particle is gbest $_{u}$; then go to Step 2 .

Step 2. Define the maximum speed particle $V_{\max }$ and the minimum speed $V_{\min }$ in $U \in R^{27}$; let $V_{\max }=\left(u_{\max }-u_{\min }\right) / 5$ and the minimum speed $V_{\min }=-V_{\max }$; then go to Step 3 .

Step 3. Generate num particles randomly in $U \in R^{27}$ between $V_{\min }$ and $V_{\max }$; denote the speed and the position of the $i$ th particle by $v^{i}=\left(v_{1}^{i}, v_{2}^{i}, \ldots, v_{27}^{i}\right)$ and $u^{i}=\left(u_{1}^{i}, u_{2}^{i}, \ldots, u_{27}^{i}\right)$; then go to Step 4 .

Step 4. Compute $J\left(u^{i}\right)$ of the IP for $i=1,2, \ldots$, num. If $J\left(u^{i}\right) \leq$ pbest, then pbest $=J\left(u^{i}\right)$. If pbest $\leq$ gbest, then gbest $=$ pbest; then go to Step 5 .

Step 5. Let $m=m+1$; if $m>T_{\max }$, then terminate the algorithm; otherwise, update the speed and the position of the particle according to the following formula:

$$
\begin{aligned}
v_{j}^{i}(m+1)= & w(m) v_{j}^{i}(m)+c_{1} r_{1 j}\left(\text { pbest }_{u j}^{i}(m)-u_{j}^{i}(m)\right) \\
& +c_{2} r_{2 j}\left(\text { g best }_{u j}(m)-u_{j}^{i}(m)\right), \\
u_{j}^{i}(m+1)= & u_{j}^{i}(m)+v_{j}^{i}(m+1) .
\end{aligned}
$$

In the above equation, $i=1,2, \ldots$, num, $j=$ $1,2, \ldots, 27, v_{j}^{i}(m), u_{j}^{i}(m)$ denote the speed and the position of the $i$ th particle in the $m$ th iteration, and $r_{1 j}$ and $r_{2 j}$ denote the random number in the interval $(0,1)$. Meanwhile, the expression of $w$ is

$$
w=(w s-w e-d 1) * \exp \left(\frac{1}{1+d 2 *(m-1) / T_{\max }}\right) .
$$

Then go to Step 4 .

Algorithm 2. Compute $J(u)$ using the following algorithm.

Step 1 (initialization). The step size is $h, h h$ and the initial time is $t$; go to Step 2 .

Step 2. For $u_{1}^{i}, u_{2}^{i}, \ldots, u_{27}^{i}$, solve the equations according to the trapezoidal method; if $x_{i}$ is in the allowable range, compute SSD; otherwise, return SSD = 10000; then go to Step 3.

Step 3. Let $n_{p}=0$ and flag $=0$; then go to Step 4 .

Step 4. Disturb $u_{i}\left(i \in I_{27}\right)$. Let disturbed value $\Delta v^{i}=u^{i}+$ $\operatorname{rand}(0.8 * u i, 1.2 * u i)$.

Step 5. Solve the equations according to the trapezoidal method for the parameters $u^{i}$ and $v^{i}$, respectively. Denote the
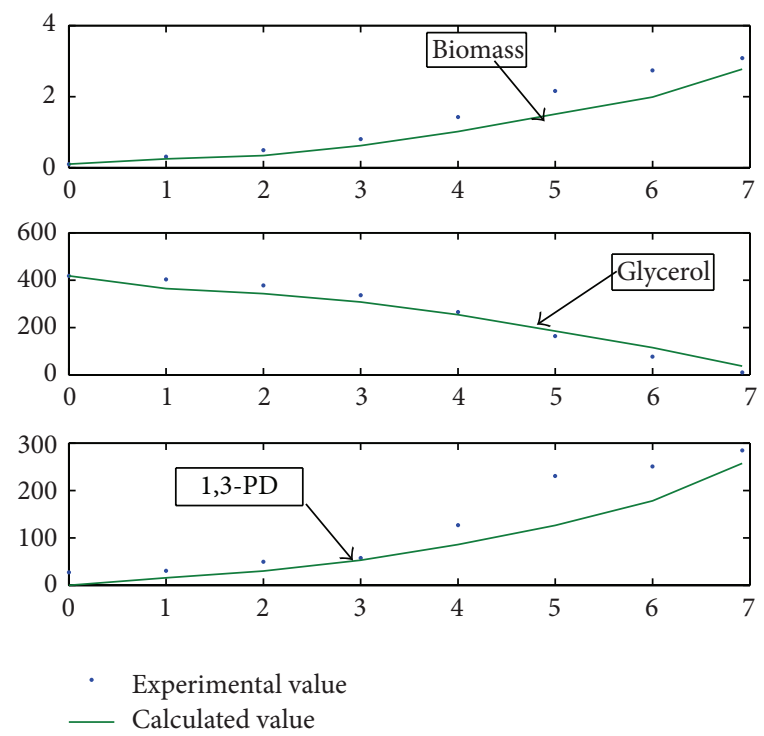

Figure 2: Comparison of extracellular concentration between computational results and the experimental data.

calculated value by $x_{k}$ and $x_{k 1}$. For $k=1, \ldots, 8$, if $x_{k} \in$ $\left[x_{k *}, x_{k}^{*}\right], x_{k 1} \in\left[x_{k *}, x_{k}^{*}\right]$, let flag $=$ flag +1 and compute MSD; otherwise, return MSD $=10000$.

Step 6. Let $n_{p}=n_{p}+1$. If $n_{p} \leq 3000$, then go to Step 4; otherwise, go to Step 7.

Step 7. Compute $\mathrm{MSD}_{\mathrm{av}}=\mathrm{MSD} /$ flag and then stop.

According to the above algorithm, the parameters chosen in the above method are as follows: num $=50, c 1=2.0$, $c 2=2.0, w s=0.9, w e=0.4, T_{\max }=1000, d 1=0.2, d 2=0.7$, $h=1 / 3600$, the initial time $t=0.0$, and $W_{\mathrm{ad}}=[0.01,15] *$ $[15,2039] *[0,1036] *[0,1026] *[0,360.9] *[0,2000] *$ $[0,80] *[0,1000]$. The identification model IP includes 27 variables.

Using the above algorithm, we obtain that the optimal parameters are listed as shown in Table 3.

Figure 2 denotes the comparisons about the experimental value and the computational value of biomass, glycerol, and 1,3-PD, respectively, where the real lines denote computational curves and the scatter grams denote experimental results. The minimum value of IP is 0.837383

\section{Conclusion}

The current work deals with the problem of modelling and system identification of the dynamical system in batch culture but there are many deficiencies in our work. In a future work, on the one hand, we will consider sensitivity analysis of kinetic parameters in our model, so as to reduce the number of these model parameters to be identified and concentrate on identifying these relatively sensitive parameters; on the other hand, we will consider three transmembrane ways to confirm 
TABLE 3: Optimal dynamical parameter vector.

\begin{tabular}{|c|c|c|c|c|c|c|}
\hline$u_{1}^{* *}$ & $u_{2}^{* *}$ & $u_{3}^{* *}$ & $u_{4}^{* *}$ & $u_{5}^{* *}$ & $u_{6}^{* *}$ & $u_{7}^{* *}$ \\
\hline 60 & 4.0012 & 300 & 100 & 2.20094 & 62.971 & 7.5 \\
\hline$u_{8}^{* *}$ & $u_{9}^{* * *}$ & $u_{10}^{* *}$ & $u_{11}^{* * *}$ & $u_{12}^{* *}$ & $u_{13}^{* *}$ & $u_{14}^{* *}$ \\
\hline 50 & 1.29593 & 2000 & 18.0101 & 253.832 & 32.0629 & 0.768834 \\
\hline$u_{15}^{* *}$ & $u_{16}^{* *}$ & $u_{17}^{* *}$ & $u_{18}^{* * *}$ & $u_{19}^{* *}$ & $u_{20}^{* * *}$ & $u_{21}^{* *}$ \\
\hline 13.4747 & 28.6098 & 88.9932 & 2.53822 & -0.780765 & 4.5 & 0.009 \\
\hline$u_{22}^{* *}$ & $u_{23}^{* *}$ & $u_{24}^{* *}$ & $u_{25}^{* *}$ & $u_{26}^{* *}$ & $u_{27}^{* *}$ & \\
\hline 29.4872 & 11.9978 & 40.6361 & 9.02496 & 1.95709 & 3.01177 & \\
\hline
\end{tabular}

which one is the most appropriate one in this improved model.

\section{Conflict of Interests}

The authors declare that there is no conflict of interests regarding the publication of this paper.

\section{Acknowledgments}

This work was supported by the National Natural Science Foundation for the Youth of China (Grant no. 11301051), 863 Program (Grant no. 2007AA02Z208), 973 Program (Grant no. 2007CB714304), and the National Natural Science Foundation of China (Grants nos. 10671126 and 10871033).

\section{References}

[1] A. Reimann, H. Biebl, and W.-D. Deckwer, "Production of 1,3propanediol by Clostridium butyricum in continuous culture with cell recycling," Applied Microbiology and Biotechnology, vol. 49, no. 4, pp. 359-363, 1998.

[2] T. Homann, C. Tag, H. Biebl, W.-D. Deckwer, and B. Schink, "Fermentation of glycerol to 1,3-propanediol by Klebsiella and Citrobacter strains," Applied Microbiology and Biotechnology, vol. 33, no. 2, pp. 121-126, 1990.

[3] H. Biebl, K. Menzel, A.-P. Zeng, and W.-D. Deckwer, "Microbial production of 1,3-propanediol," Applied Microbiology and Biotechnology, vol. 52, no. 3, pp. 289-297, 1999.

[4] K. Menzel, A.-P. Zeng, and W.-D. Deckwer, "High concentration and productivity of 1,3-propanediol from continuous fermentation of glycerol by Klebsiella pneumoniae," Enzyme and Microbial Technology, vol. 20, no. 2, pp. 82-86, 1997.

[5] A.-P. Zeng and W.-D. Deckwer, "A kinetic model for substrate and energy consumption of microbial growth under substratesufficient conditions," Biotechnology Progress, vol. 11, no. 1, pp. 71-79, 1995.

[6] Z. L. Xiu and A. P. Zeng, "Mathematical modelling of kineti cs and research on multiplicity of glycerol bioconversion to 1,3 propanediol," Journal of Dalian University of Technology, vol. 40, pp. 428-433, 2000.

[7] C. Gao, E. Feng, Z. Wang, and Z. Xiu, "Parameters identification problem of the nonlinear dynamical system in microbial continuous cultures," Applied Mathematics and Computation, vol. 169, no. 1, pp. 476-484, 2005.

[8] Y. Q. Sun, W. T. Qi, H. Teng, Z. L. Xiu, and A. P. Zeng, "Mathematical modeling of glycerol fermentation by Klebsiella pneumoniae: concerning enzyme-catalytic reductive pathway and transport of glycerol and 1,3-propanediol across cell membrane," Biochemical Engineering Journal, vol. 38, no. 1, pp. 22-32, 2008.

[9] J. Wang, J. Ye, E. Feng, H. Yin, and Z. Xiu, "Modeling and identification of a nonlinear hybrid dynamical system in batch fermentation of glycerol," Mathematical and Computer Modelling, vol. 54, no. 1-2, pp. 618-624, 2011.

[10] C. X. Gao, Z. T. Wang, E. M. Feng, and Z. L. Xiu, "Parameter identification and optimization of process for bio-dissimilation of glycerol to 1,3-propanediol in batch culture," Journal of Dalian University of Technology, vol. 46, pp. 771-774, 2006.

[11] L. Wang, J. Ye, E. Feng, and Z. Xiu, "An improved model for multistage simulation of glycerol fermentation in batch culture and its parameter identification," Nonlinear Analysis: Hybrid Systems, vol. 3, no. 4, pp. 455-462, 2009.

[12] J. Song, J. Q. Lin, Y. Jin et al., "A new population growth model based on the time dependent changes of the specific growth rate," Microbiology, vol. 34, pp. 836-838, 2007.

[13] Z. Bing, L. B. Jia, and Y. Lei, "Progress of biological robustness," Acta Biophysica Sinica, vol. 23, pp. 357-363, 2007.

[14] H. Kitano, "Biological robustness," Nature Reviews Genetics, vol. 5, no. 11, pp. 826-837, 2004.

[15] H. Kitano, "Towards a theory of biological robustness," Molecular Systems Biology, vol. 3, article 137, 2007.

[16] J. Wang, J. Ye, E. Feng, H. Yin, and B. Tan, "Complex metabolic network of glycerol fermentation by Klebsiella pneumoniae and its system identification via biological robustness," Nonlinear Analysis: Hybrid Systems, vol. 5, no. 1, pp. 102-112, 2011.

[17] Y. Zhang, E. Feng, and Z. Xiu, "Robust analysis of hybrid dynamical systems for 1,3-propanediol transport mechanisms in microbial continuous fermentation," Mathematical and Computer Modelling, vol. 54, no. 11-12, pp. 3164-3171, 2011.

[18] J. Kennedy and R. Eberhart, Swarm Intelligence, Academic Press, New York, NY, USA, 2001.

[19] H. Kitano, "Violations of robustness trade-offs," Molecular Systems Biology, vol. 6, no. 1, article 384, 2010.

[20] M. Perc and M. Marhl, "Sensitivity and flexibility of regular and chaotic calcium oscillations," Biophysical Chemistry, vol. 104, no. 2, pp. 509-522, 2003.

[21] M. Perc and M. Marhl, "Noise enhances robustness of intracellular $\mathrm{Ca}^{2+}$ oscillations," Physics Letters A, vol. 316, no. 5, pp. 304310, 2003.

[22] N. Barkai and S. Leibler, "Robustness in simple biochemical networks," Nature, vol. 387, no. 6636, pp. 913-917, 1997.

[23] U. Alon, M. G. Surette, N. Barkai, and S. Leibler, "Robustness in bacterial chemotaxis," Nature, vol. 397, no. 6715, pp. 168-171, 1999.

[24] D. S. Callaway, M. E. J. Newman, S. H. Strogatz, and D. J. Watts, "Network robustness and fragility: percolation on random graphs," Physical Review Letters, vol. 85, pp. 5468-5471, 2000. 


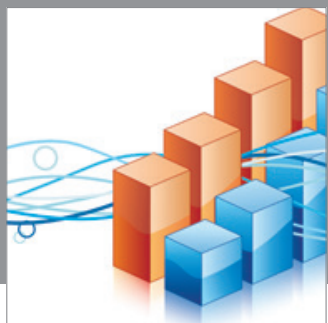

Advances in

Operations Research

mansans

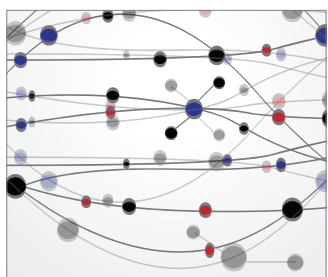

The Scientific World Journal
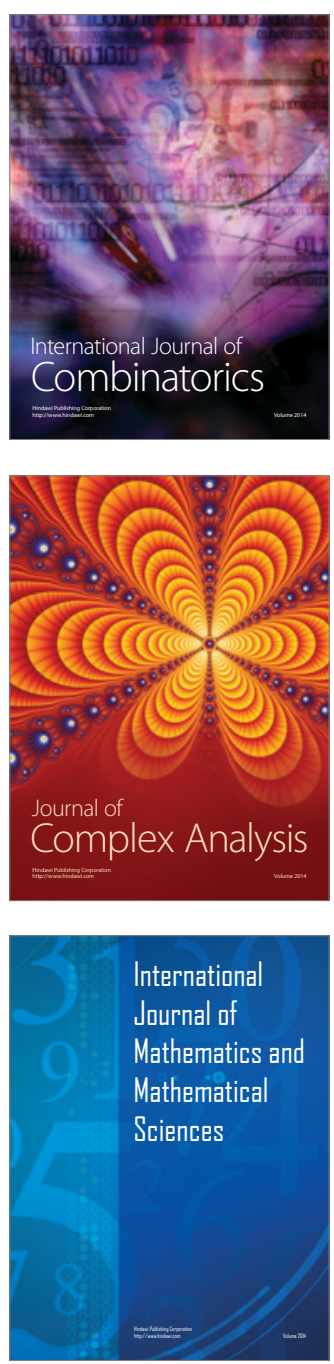
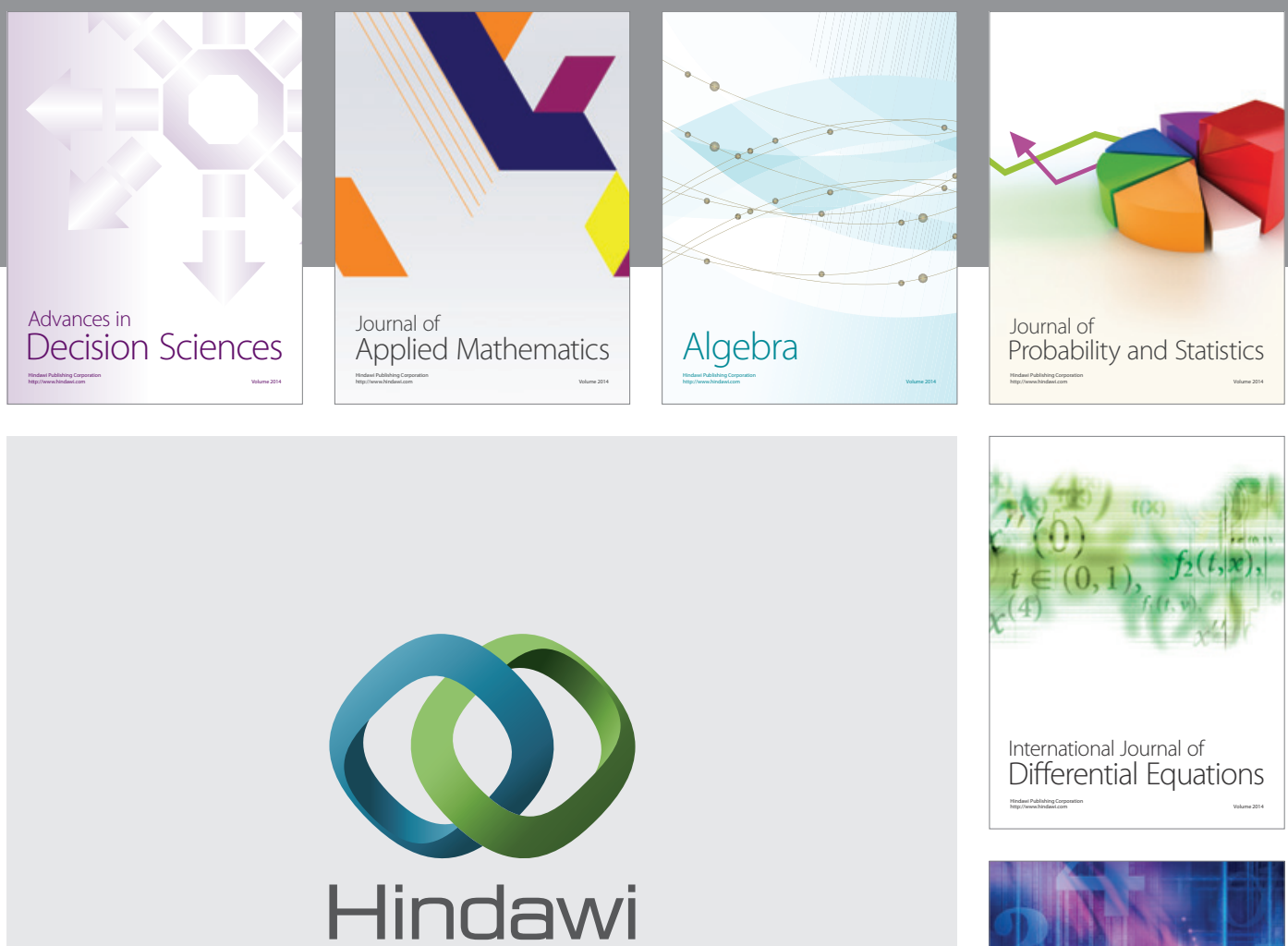

Submit your manuscripts at http://www.hindawi.com
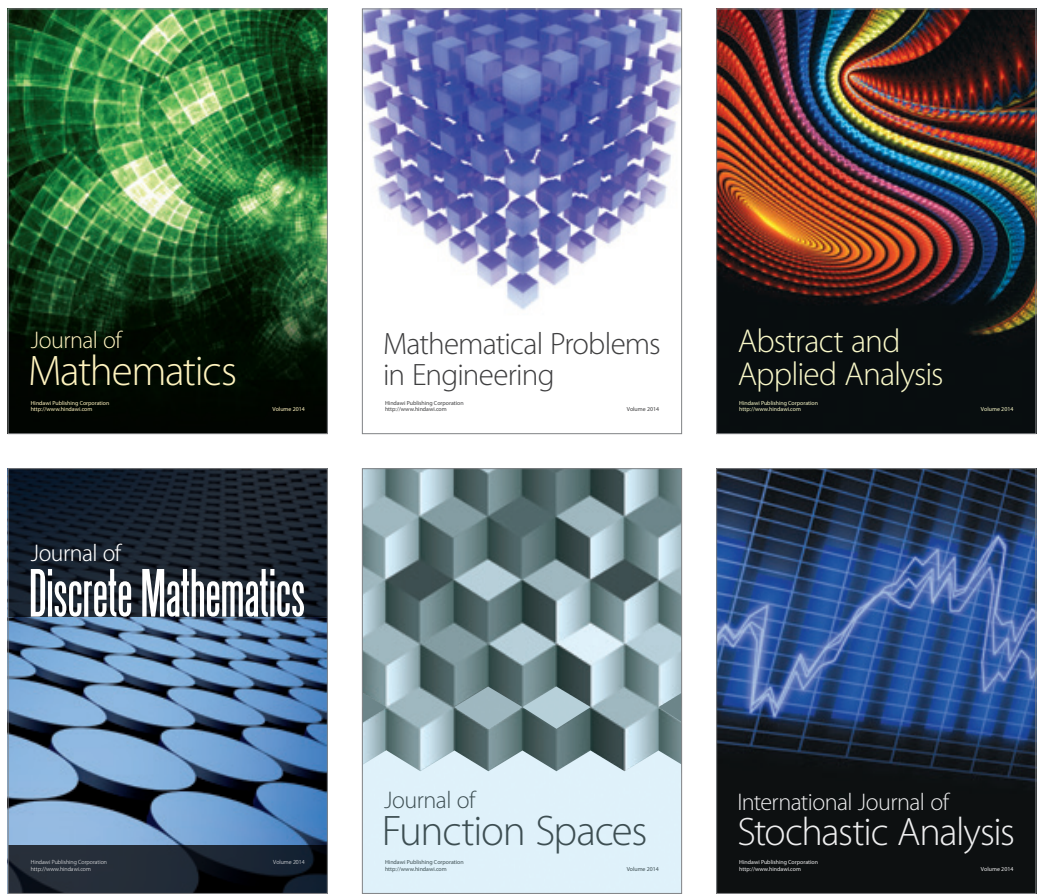

Journal of

Function Spaces

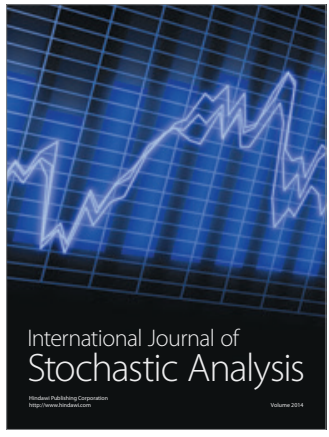

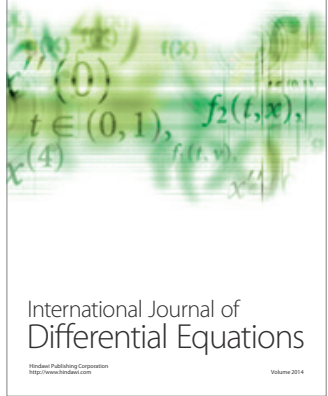
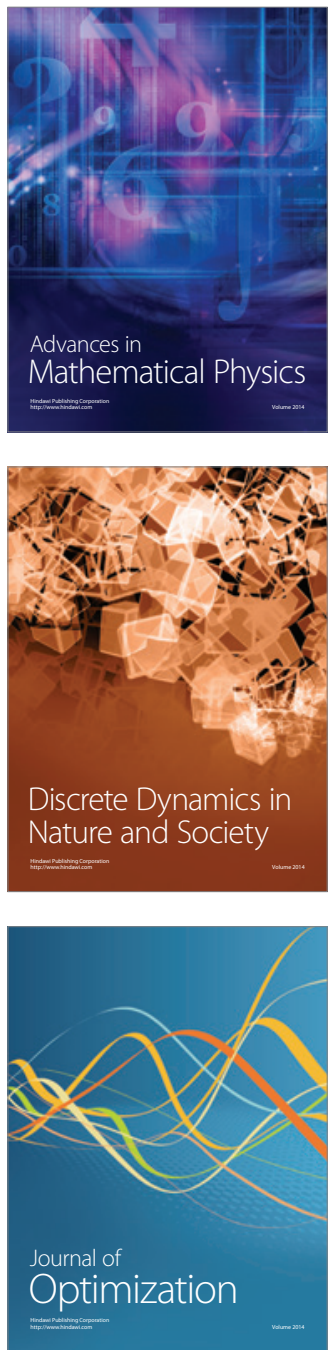